Published in final edited form as:

Prostate. 1999 February 15; 38(3): 237-245.

\title{
Citrate in the Diagnosis of Prostate Cancer
}

\author{
L.C. Costello ${ }^{1, *}$, R.B. Franklin ${ }^{1}$, and P. Narayan ${ }^{2}$ \\ ${ }^{1}$ Cellular and Molecular Biology Section, Department of Oral-Cranial Biological Sciences, Dental \\ School, University of Maryland, Baltimore, Maryland ${ }^{2}$ Division of Urology, College of Medicine, \\ University of Florida, Gainesville, Florida
}

\section{Abstract}

BACKGROUND-One of the major current problems involved in prostate cancer (PCa) is the unavailability of sensitive, accurate, and preferably noninvasive procedures for the diagnosis of PCa. Moreover, procedures are needed which will permit the early detection, staging, location, and estimation of the volume of malignancy, and preferably a mapping of the prostate for followup of progression and regression of the malignancy.

METHODS-The unique citrate relationships of the prostate, coupled with recent developments and technological advancements in magnetic resonance spectroscopy (MRS) for the in situ determination of citrate levels, now provides an excellent diagnostic procedure which can achieve all these goals. There exist strong, compelling basic and clinical studies in support of the employment of 1H MRS measurements of citrate and other associated metabolites in the diagnosis of PCa.

RESULTS-This review provides the background leading to the current status of MRS citrate analysis, summarizes the data from clinical trials, and describes the applications of the procedure for the diagnosis of PCa and follow-up of patients. The use of MRS studies in defining the functional, as well as pathological relationships of the prostate, is also discussed.

CONCLUSIONS-This review is intended to be informative to the prostate- and oncologyinterested community, and, hopefully, to engender much-needed interest and support in future research regarding the prostate relationships described in this report.

\section{Keywords}

prostate cancer; citrate metabolism; magnetic resonance spectroscopy; zinc; benign prostatic hyperplasia

\section{INTRODUCTION}

Prostate cancer (prostate adenocarcinoma, $\mathrm{PCa}$ ) is the most common nonskin cancer in American men, with about 250,000 new cases annually in recent years. PCa is the cause of

${ }^{*}$ Correspondence to: Dr. Les Costello, Department of Oral-Cranial Biological Sciences, Dental School, University of Maryland, 666 W. Baltimore St., Baltimore, MD 21201. lcc001@ dental.umaryland.edu. 
about 40,000 deaths annually, only second to lung cancer in number of deaths in males due to cancer. The survival and successful treatment of PCa patients are dependent upon the early diagnosis of $\mathrm{PCa}$, and the ability to monitor the progression and regression of malignancy. Presently the combination of digital rectal examination and prostate-specific antigen (PSA) testing is the principal routine diagnostic procedure. Histopathological examination of biopsy material and cross-sectional imaging procedures are also employed in diagnostic and staging procedures. However, there are several limitations and problems with current diagnostic procedures. In all cases, positive identification of PCa only becomes evident when malignancy has been established; this is often after the malignant cells have extended and metastasized beyond the capsular region of the prostate. Staging tests are inaccurate $60 \%$ of the time and are unable to estimate accurately the cancer volume within or outside the capsule of the prostate. Moreover, the tracking of the progression and regression of malignancy, especially following treatment, has proven to be difficult. For these and other reasons, there is an urgent need for the development of new and alternative diagnostic procedures and markers for the identification and management of PCa patients.

"The authors propose that measurable biochemical changes may occur in malignant transformation of the human prostate long before a definitive histological diagnosis can be made by the pathologist. If such a premise can be substantiated, biochemical detection of malignancy may become possible in the early, formative stages of prostatic carcinoma when no clinical signs are evident." This was the prophetic statement made in 1964 by Cooper and Farid as they proceeded to present their citrate studies $[1,2]$. As this review will show, strong compelling evidence has accumulated over the years which supports the use of prostate citrate measurements as a reliable identification of PCa. Evidence exists that the biochemical assaying of citrate levels will provide for the earliest detection of $\mathrm{PCa}$. Moreover, recent advances in magnetic resonance spectroscopy (MRS), when applied to prostate citrate measurements, provide a promising noninvasive procedure for detecting, staging, and tracking PCa. Despite this accumulated evidence, the importance and use of citrate measurements in PCa have been largely ignored by most researchers and clinicians involved in prostate disease. The purpose of this report is to review the literature in support of the diagnostic role of citrate, to identify the current status of noninvasive MRS procedures for citrate diagnosis and staging of $\mathrm{PCa}$, and to discuss the immediate and future advances required to employ these procedures for common clinical use. We hope this will heighten the awareness of and interest in this most promising advancement.

\section{THE CITRATE RELATIONSHIP IN PCa}

It has long been recognized that the normal human prostate gland uniquely accumulates and secretes extraordinarily high citrate levels (Table I). For detailed reviews of the metabolic relationships and hormonal regulation of prostate citrate, we refer the reader to our previous publications [3-8]. In 1954, Cooper and Imfeld [9] first reported the citrate relationship in malignant vs. benign prostatic tissue. Their studies revealed that the citrate levels of benign tissue (benign prostatic hyperplasia, BPH), as with normal prostate, were extremely high. In contrast, the citrate levels of malignant prostate tissue were significantly lower than the benign prostate levels. Marberger et al. [10] reported that metastatic tissue derived from PCa contained very low citrate levels. These observations were corroborated and expanded by 
Cooper and Farid $[1,2]$. They reported that the citrate levels in "early carcinoma" were significantly lower $(-36 \%)$ than in BPH; and in "advanced carcinoma" the citrate levels were drastically lower $(-86 \%)$. When they referenced citrate values against lactate values (which remained constant), the citrate changes were even more dramatic. There was no overlap in the lactate/citrate ratios of either early or advanced PCa vs. BPH. They concluded that citrate changes " ... are characteristic, reproducible, and useful in the biochemical differentiation of benign and malignant prostate disease"; and they added, "It is possible that such biochemical observations may prove of clinical value in the early diagnosis of prostate carcinoma." Of additional importance was their premise " ... that biochemical alteration of the neoplastic prostate ... may even precede histologic evidence of malignancy." These important observations remained dormant until 1978, when Costello et al. [3] confirmed the decrease in prostate citrate levels of $\mathrm{PCa}$ vs. $\mathrm{BPH}$, and established that the changes in citrate were even more pronounced when related to the glandular component of the tissue sample, since stromal tissue contained very little citrate. Collectively, the biochemical studies described above constituted the fundamental evidence that reduced citrate levels provide an important biochemical marker which is effective in the differentiation of $\mathrm{PCa}$ from normal and BPH prostate and in the identification of prostate malignancy. Despite the availability of this information, and the urgings in review articles [3-8], the importance of citrate relationships in the diagnosis of $\mathrm{PCa}$ as well as other implications continued to be largely ignored by the prostate clinical and research community. The exception to this was a small group of investigators with expertise in MRI techniques who, beginning around 1988, recognized the potential of prostate citrate analysis as a diagnostic tool.

\section{MRS MEASUREMENT OF CITRATE: HISTORICAL PERSPECTIVE}

The biochemical studies described above required the acquisition of prostate tissue (needle biopsy, TURP, prostatectomy) for tissue citrate analysis. A major advancement for the application of the citrate relationship in PCa would be achieved by a noninvasive procedure permitting the in situ, direct determination of citrate in the prostate. Sillerud et al. [11] and Halliday et al. [12] successfully applied carbon (13C) MRS technology measurement of prostate citrate levels and confirmed the characteristic decrease in citrate levels in PCa. Because of inherent difficulties with carbon MRS measurements (see Schiebler et al. [13] for an excellent critical review of MRI methods applied to the prostate), attention was directed toward the use of $1 \mathrm{H}$ MRS determination of citrate and other metabolic intermediates. Beginning in 1989, Narayan et al. [14-18], developed the technique of performing human MRS studies using an endorectal coil. Initial feasibility studies with small surface coils were performed on Dunning rat prostate cancers grown on the flank, which led to the subsequent development of single- and double-tuned endorectal coils. These were tested in canines with normal and malignant prostates to determine safety as well as feasibility. Subsequently, several generations of endorectal coils were developed and tested in healthy human volunteers and in patients with BPH and PCa. The human studies demonstrated that endorectal 1H MRS analysis of prostate citrate levels in situ could identify and differentiate $\mathrm{PCa}$ from normal and $\mathrm{BPH}$ subjects, thereby verifying the earlier biochemical analyses of reduced citrate levels in malignant prostate tissue. These studies corroborated the earlier studies of Schnall et al. [19]. Applying 1H MRS spectroscopy to 
resected prostate tissue samples, Fowler et al. [20] and Cornel et al. [21] corroborated the findings of decreased citrate in PCa as observed in situ.

Currently, both rigid and expandable endorectal coils are available for performing spectroscopic imaging of the human prostate in situ, with each having inherent advantages and disadvantages. The latter are advantageous in that they can be combined with an external pelvic phased array coil in order to provide MRI coverage of the prostate and pelvis. They are disposable and commercially available. Additionally, the methodology for assessing citrate and other metabolites in the entire prostate using spectroscopy has been developed. The methodology is called MR spectroscopic imaging (MRSI) and is a marriage of MRI with MRS. MRSI produces arrays of contiguous spectra in one, two, or three dimensions from volumes of tissue as small as $0.24 \mathrm{cc}[22,23]$. This metabolic information can be subsequently overlaid on the corresponding anatomic image (MRI).

A major problem in assessing the presence and spatial extent (volume) of prostate cancer by MRI alone is a lack of specificity in identifying prostate cancer. This lack of specificity is due to factors other than cancer (e.g., postbiopsy hemorrhage, prostatitis, and therapy) that alter or mask the MR images of the peripheral zone used as an indicator of cancer. 1H MRS measurement of citrate and other metabolites has demonstrated high specificity in discriminating prostate cancer from surrounding regions of normal peripheral zone [23].

In situ 1H MRS of the human prostate has been limited by technical issues associated with data acquisition (suppression of water and lipids) and data processing. Currently there are no commercially available packages for the processing and display of volume MRI/MRSI data. However, the resolution of these problems, and combined MRI and MRSI for citrate measurements, offer a great potential for the noninvasive identification of prostate cancer.

\section{CLINICAL APPLICATION OF IN SITU MRS MEASUREMENTS OF CITRATE IN THE DIAGNOSIS AND STAGING OF PCa}

The relationships and developments described above set the stage for a noninvasive diagnostic procedure for the identification of $\mathrm{PCa}$ in patients. Although the actual tissue citrate level per se is significantly lower in PCa than in normal and/or BPH tissue, the variability of the citrate concentration is somewhat affected by the composition of the field or tissue sample being analyzed. To minimize such variations, citrate was referenced to choline levels or choline + creatine levels [22-24]. The use of such ratios also minimized geometry factors in the comparison of spectroscopic voxels (tissue volumes) in the same examination or between examinations. In contrast to the decrease in citrate, choline levels are generally increased in malignant areas, which enhances the metabolic discrimination of cancer from normal peripheral zone tissues. Use of these refinements and combination of three-dimensional (3-D) MRSI with MR imaging (Figs. 1, 2) have been extremely successful in the identification of PCa, in differentiating PCa from normal and BPH prostate, and in establishing the zonal differences in citrate production [23-25].

Figure 3 summarizes some collective data derived from three separate and independent reports $[23,24,26]$ which are worthy of careful scrutiny and discussion. The information in 
these three reports employed noninvasive endorectal MRS technology with slightly different variations in expressing the changes in citrate composition.

Several important relationships are now established (Figs. 1-3). Clearly, zonal differences exist in the normal human prostate. The normal peripheral zone is the region principally involved in citrate production, as represented by the highest citrate levels. In contrast, the central gland (central zone and transition zone) and periurethral region contain significantly lower citrate levels. It is evident, also, that glandular BPH is characterized by an increase in citrate levels in the nodular regions of the central zone and transition zones (Figs. 1, 2). In all three studies based on citrate relationships, cancer patients could be differentiated clearly from BPH and normal subjects (Fig. 3). This is especially evident if citrate measurements are targeted at the peripheral zone, where there is virtually no overlap between the citrate levels of PCa patients and of nonmalignant patients. When coupled with MRI, 1H MRS citrate analysis could localize the malignancy and provide an accurate estimate of its volume. Particularly striking and most importantly, there exists no case in which a histopathologically confirmed malignant region retained and exhibited the typical high citrate concentration characteristic of the normal region of the peripheral zone. This confirms the view that citrate-producing prostate epithelial cells are incapable of being malignant cells, and that malignant prostate cells are citrate-oxidizing cells [6-8,27].

There is occasional overlap between the lowest citrate levels in BPH and the highest citrate levels in PCa. This has raised some concern regarding the clinical interpretation of such data [22-24,28]. However, if citrate measurements are targeted at the peripheral zone, the central zone BPH values are eliminated. We suggest that a low citrate value for the peripheral zone or central zone, observed in subjects with confirmed glandular BPH but no clinical manifestations of $\mathrm{PCa}$, would be indicative of the existence of very early undetected malignancy and/or a premalignant condition (discussed below). In Figure 3, those BPH subjects with low central zone citrate levels would be potential cancer patients. Also, MRS detection of low citrate in the peripheral zone of "healthy" subjects could be indicative of a premalignant condition. Such individuals should be followed closely for the onset of clinical appearance of malignancy.

Costello et al. [3] reported that high citrate levels in the prostate were due to the glandular component, and that stromal tissue contained the low citrate values typical of soft tissues in general. Despite this, in BPH, citrate levels are higher than in PCa, even in the presence of high stromal content, as long as some glandular tissue is present. Moreover, neighboring nonprostatic epithelial tissue, as well as nonmalignant prostate regions, which are virtually devoid of peripheral zone glandular components, would be expected to exhibit low citrate levels. Under these conditions, the presence of malignant loci in these regions of normally low citrate content would not be identified by MRS detection of citrate levels. However, malignancy in these areas will generally follow the onset of malignancy in the peripheral zone and will represent only about $30 \%$ of PCa cases.

The observations concerning zonal differences warrant some additional comment. Based on ontogenetic and homologous relationships, Costello and Franklin [4] had proposed that the peripheral zone was the major citrate-producing region, and that the central zone was not 
principally involved in citrate production. MRS studies now confirm this functional and metabolic regional difference. Lowry et al. [29] reported that the normal peripheral zone citrate level is fourfold higher than in the central zone. This emphasizes and supports the supposition that there exist different functional glandular epithelial cells, probably of different embryonic origins, associated with the various regions of the prostate [4-8]. The embryogenesis of the lateral zone is from the urogenital sinus, whereas the central zone is derived from the Wolffian duct [5,30,31]. It is now evident that glandular BPH involves the invasion and proliferation of citrate-producing epithelial cells into the central zone (Figs. 1, 2). In contrast, $\mathrm{PCa}$ involves a metabolic transformation from citrate-producing nonmalignant cells to citrate-oxidizing malignant cells, predominantly in the peripheral zone (especially in the lateral region), as earlier proposed [4].

Presently, citrate levels indexed with either choline or choline + creatine appear to be preferable for the identification of PCa (Fig. 3). Liney et al. [26] obtained impressive results when coupling the absolute citrate concentration with MRI. Nevertheless, improvements in measurements should be pursued. We found it striking that the early studies of Cooper and Farid [1,2], which employed the citrate/lactate ratio, were so remarkable in successfully differentiating malignant from nonmalignant prostate tissue samples: this, in spite of the relatively crude assay procedures employed and the inherent variability of the excised tissue samples. The key aspect of those measurements was the fact that lactate levels remained relatively constant regardless of the pathology and histological variability of the samples. Cornel et al. [21], using 1H MRS, compared the relative integrated peak ratios of several intermediates for the differentiation of malignant from nonmalignant prostate samples. They found that the citrate/lactate ratio provided the most discriminatory parameter, even better than the citrate/choline and citrate/creatine ratios. There was no overlap in the citrate/lactate ratios, with the mean ratio for $\mathrm{BPH}$ being 10 times greater than for PCa tissue samples. Fowler et al. [20] reported that MRS revealed very little change in alanine in PCa vs. BPH vs. mixed prostate extracts. The citrate/alanine ratio for PCa was significantly lower than for BPH. However, it must be recognized that in the above studies, extract preparations of resected prostate samples were employed. The complex spectra of in vivo MRS analyses obscured the clear identification of these and other intermediates. The essential issue, currently, is whether changing citrate referenced to a constant component or changing citrate enhanced with other changing components will represent the best index of PCa. Conceivably, intermediates which remain constant and can be resolved by in situ MRS will be identified as references for citrate changes.

\section{MRS CITRATE ANALYSIS IN THE FOLLOW-UP OF PROSTATE CANCER}

As important as the diagnosis of $\mathrm{PCa}$ is a procedure which will permit the follow-up of patients after PCa has been identified. The progression or regression of the malignancy over time and subsequent to treatment is extremely important to ascertain in the management of $\mathrm{PCa}$. Current diagnostic procedures such as PSA, radiological techniques, and histopathological examination of biopsy samples are inadequate. Recent advances in combining MRI and 3-D 1H MRS citrate analysis in situ appear to provide an extremely accurate, noninvasive procedure for following the progression or regression of malignancy in the PCa patient. In studies of PCa patients before and after cryosurgery [32], the presence 
and location of residual cancer by combined MR imaging and spectroscopic analysis were accurately determined. A contiguous spectroscopic record of the prostate of each patient was obtained and volumes of cancer as small as $0.24 \mathrm{ccm}$ were detected by citrate analysis. 3-D MRS examination was far superior and more sensitive than other methods (biopsy examination, ultrasound, or MRI) in detecting residual and recurrent cancer loci and in differentiating PCa from BPH and necrotic areas. Thus, this procedure can provide a permanent record of the normal and pathological map of the prostate gland of a patient, which can be subsequently followed as a continuous history of the patient (Fig. 2).

\section{THE PREMALIGNANT STATE AS CHARACTERIZED BY CITRATE CHANGES}

The value of following changes in tissue citrate levels in the diagnosis and tracking of PCa is apparent from the preceding discussion. However, there are additional implications to be derived from the collective studies cited above. The consistency of the decreased citrate associated with malignant prostate is striking. The decrease is evident despite large variations in tissue preparations and assay procedures. The citrate change in PCa is evident in biopsy samples, in TURP samples, in prostatectomy samples, and in situ. The citrate change is apparent even with large variations in the histological composition of the sample. It is obvious that the ratio of stroma to glandular components of the many samples represented in the composite of all the reported studies must have varied considerably. The decreased citrate in $\mathrm{PCa}$ is detectable despite the admixture of normal, benign, and malignant epithelial cell populations within the sample being analyzed. The MRS studies appear to corroborate this premise in that the volumes (voxels) analyzed often include significant amounts of nonmalignant glandular tissue but still exhibit decreased citrate signal when PCa has been identified. With very little exception [28], virtually all reports to date consistently demonstrate a significant decrease in citrate content of prostate with demonstrable malignancy. As shown in Figure 3, there exists virtually no observation of malignant tissue which has retained the high citrate level characteristic of normal and $\mathrm{BPH}$ tissue!

From these relationships, one must conclude that, by the time the onset of malignancy is established, a significant decrease in citrate levels is apparent, particularly in the region of the peripheral zone. One would normally expect a proportional gradation of citrate changes as the ratio of malignant vs. nonmalignant cells increases. If so, a large population of identifiable malignant cells would be required to effect a detectable change. However, such significant citrate changes are evident even when minimal malignant foci are surrounded by predominantly normal and/or glandular BPH components. Assume that a section of resected prostate tissue or a voxel selected for MRS measurements contains, by histopathological examination, about $10 \%$ malignant cells with the remainder being comprised of a combination of normal and BPH glandular epithelium and some stroma. In the absence of any malignancy, the citrate level would be about $20,000 \mathrm{nmol} / \mathrm{g}$ tissue. The expectation would be that in the presence of $10 \%$ malignancy, the citrate level of the sample would be about $18,000 \mathrm{nmol} / \mathrm{g}$. Such a change would be extremely difficult to detect by tissue extract analysis or even by MRS analysis. We were impressed that, even when malignancy has developed to the extent of only $10 \%$ of the tissue examined, the decrease in citrate is dramatic, readily demonstrable, and far greater than $10 \%$ [3]. This is also evident in the 
report of Schiebler et al. [28], which observed the low MRS citrate signal in a tissue sample which contained minimal malignant glands in a field dominated by normal glands.

What is the basis for this relationship? The most plausible explanation is that a large population of glandular epithelial cells which do not produce citrate must exist once the onset of malignancy has been established. Normal and BPH epithelial cells are citrateproducing cells which have a limited capability to oxidize citrate. In contrast, malignant prostate cells are citrate-oxidizing cells and do not accumulate citrate. This is the metabolic transformation associated with the neoplastic development of malignant prostate cells. This metabolic transformation must exist in a large population of cells which are not histopathologically identified as malignant cells, and which retain the organizational appearance of normal or BPH epithelial cells. The existence of a premalignant stage involved in the development and progression of malignancy has been proposed (Fig. 4) $[7,8]$. This would be the reason why the biochemical changes associated with malignancy precede the histopathological changes. This probably accounts, at least in part, for the reported absence of a proportional decrease in citrate as correlated with the Gleason grade [20].

\section{RELATIONSHIP BETWEEN ZINC AND CITRATE}

Like citrate, zinc accumulates in uniquely high levels in normal and BPH prostate.

Moreover, it is now well-established that PCa is characterized by a marked decrease in zinc along with the decrease in citrate. Virtually all the relationships described for citrate are applicable to zinc. Costello and Franklin [8] reviewed the role of zinc in the metabolism of citrate in normal and malignant prostate cells. It is now evident that the accumulation of zinc in normal and BPH epithelial cells results in the inhibition of citrate oxidation and in the accumulation of citrate. In malignant prostate cells the ability to accumulate zinc is lost so that citrate oxidation occurs, resulting in the decreased citrate level associated with PCa. Thus the decrease in zinc is a prerequisite to the decrease in citrate level. This is consistent with earlier reports $[33,34]$ that decreased zinc levels could be a marker for the early identification of PCa. Therefore, the zinc relationship provides another parameter for the diagnosis of PCa. It might be possible to couple the detection of changes of both zinc and citrate to provide a more sensitive and reliable diagnosis of $\mathrm{PCa}$.

\section{IN SITU MRS IN ESTABLISHING THE METABOLIC RELATIONSHIPS IN THE PATHOGENESIS AND PROGRESSION OF PROSTATE MALIGNANCY}

In addition to clinical applications, MRS in situ studies have provided extremely important information regarding the nature of PCa. MRS studies essentially detect dynamic in situ changes in cellular intermediary metabolism during the malignant process. No longer can there be any question that prostate malignancy involves a major metabolic alteration in citrate-related metabolism associated with the pathogenesis of malignancy, as previously proposed [3-8]. Moreover, MRS and other studies support the hypothesis that the metabolic transformation involves alteration in the oxidation of citrate by malignant vs. non-malignant epithelial cells. These metabolic relationships are unique to the prostate and are not observed in other malignant cells. Such significant metabolic alterations will have profound effects on 
cellular intermediary energy metabolism. These relationships, once established, will aid in elucidating the pathogenesis of PCa and will provide new approaches to its treatment. MRS applications for determining altered citrate-related metabolism and the energetic implications (e.g., 31P MRS) in experimental models of prostate malignancy and in humans provide an exciting tool for future research in understanding the normal and pathological prostate.

\section{Acknowledgments}

Grant sponsor: NIH; Grant numbers: DK 28015, CA 71207, DK 42839.

We express our appreciation to Dr. John Kurhanewicz (Department of Radiology, University of California at San Francisco) for his most valuable comments in the preparation of this paper. This report and portions of the data presented were supported by NIH research grants DK 28015, CA 71207, and DK 42839 (to L.C.C. and R.B.F.).

\section{References}

1. Cooper JE, Farid I. The role of citric acid in the physiology of the prostate. A chromatographic study of citric acid cycle intermediates in benign and malignant prostatic tissue. J Surg Res. 1963; 3:112-121. [PubMed: 14022851]

2. Cooper JE, Farid I. The role of citric acid in the physiology of the prostate. Lactic/citrate ratios in benign and malignant prostatic homogenates as an index of prostatic malignancy. J Urol. 1964; 92:533-536. [PubMed: 14226486]

3. Costello, LC.; Littleton, G.; Franklin, RB. Regulation of citrate related metabolism in normal and neoplastic prostate. In: Sharma, RK.; Criss, WE., editors. Endocrine control in neoplasia. New York: Raven Press; 1978. p. 303-313.

4. Costello LC, Franklin RB. Concepts of citrate production and secretion by prostate: 1. Metabolic relationships. Prostate. 1991; 18:25-46. [PubMed: 1987578]

5. Costello LC, Franklin RB. Concepts of citrate production and secretion by prostate: 2. Hormonal relationships in normal and neoplastic prostate. Prostate. 1991; 19:181-205. [PubMed: 1946039]

6. Costello LC, Franklin RB. Citrate metabolism of normal and malignant prostate epithelial cells. Urology. 1997; 50:3-12. [PubMed: 9218011]

7. Franklin, RB.; Costello, LC. Intermediary energy metabolism of normal and malignant prostate epithelial cells. In: Naz, RK., editor. Prostate: basic and clinical aspects. New York: CRC Press; 1997. p. 115-150.

8. Costello LC, Franklin RB. The novel role of zinc in the intermediary metabolism of prostate epithelial cells and its implications in prostate malignancy. Prostate. 1998; 35:285-296. [PubMed: 9609552]

9. Cooper JF, Imfeld H. The role of citric acid in the physiology of the prostate: a preliminary report. J Urol. 1959; 81:157-163. [PubMed: 13631793]

10. Marberger H, Marberger E, Mann T, Lutwak-Mann C. Citric acid in human prostatic secretion and metastasizing cancer of prostate gland. Br Med J. 1962; 1:835-836. [PubMed: 14469565]

11. Sillerud LO, Halliday KR, Griffey RH, Fenoglia-Preiser C, Sheppard S. In vitro 13C NMR spectroscopy of the human prostate. Magn Reson Med. 1988; 8:224-230. [PubMed: 2463457]

12. Halliday KR, Fenoglio-Preiser C, Sillerud LO. Differentiation of human tumors from nonmalignant tissue by natural abundance 13C NMR spectroscopy. Magn Reson Med. 1988; 7:384411. [PubMed: 2459580]

13. Schiebler ML, Schnall MD, Pollack HM, Lenkinski RE, Toamszewski JE, Wein AJ, Whittington R, Rauschning W, Kressel HY. Current role of MR imaging in the staging of adenocarcinoma of the prostate. Radiology. 1993; 189:339-352. [PubMed: 8210358]

14. Narayan D, Vigneron DB, Jajodia CM, Anderson CM, Hedgcock MW, Tanagho EA, James TL. Transrectal probe for 1H MRI and 31P MR spectroscopy of the prostate gland. J Magn Reson. 1989; 11:209-220. 
15. Thomas MA, Narayan P, Kurhanewicz J, Jojadia P, Weiner MW. 1H MR spectroscopy of normal and malignant human prostate in vivo. J Magn Reson. 1990; 87:610-619.

16. Kurhanewicz J, Thomas A, Jajodia P, Weiner M, James TL, Vigneron D, Narayan P. 31P spectroscopy of the human prostate gland in vivo using a transrectal probe. Magn Reson Med. 1991; 22:404-413. [PubMed: 1725918]

17. Narayan P, Kurhanewicz J. Magnetic resonance spectroscopy in prostate disease: diagnostic possibilities and future developments. Prostate. 1992; 4:43-50. [PubMed: 1374177]

18. Kurhanewicz J, Dahiya R, MacDonald JM, Chang LH, James TL, Narayan P. Citrate alterations in primary and metastatic human prostate adenocarcinomas. $1 \mathrm{H}$ magnetic resonance spectroscopy and biochemical study. Magn Reson Med. 1993; 29:149-157. [PubMed: 8429778]

19. Schnall MD, Lekinski RL, Pollack HM, Imai Y, Kressel HY. Prostate MR imaging with an endorectal surface coil. Radiology. 1989; 172:570-574. [PubMed: 2748842]

20. Fowler AH, Pappas AA, Holder JC, Finkbeiner AE, Dalrymple GV, Mullins MS, Sprigg JR, Komoroski RA. Differentiation of human prostate cancer from benign hypertrophy by in vitro $1 \mathrm{H}$ NMR. Magn Reson Med. 1992; 25:140-147. [PubMed: 1375702]

21. Cornel EB, Smits GAHJ, Oosterhof GON, Karthaus HFM, Debruyne FMJ, Schalken JA, Heerschap A. Characterization of human prostate cancer, benign prostatic hyperplasia and normal prostate by 1H and 31P magnetic resonance spectroscopy. J Urol. 1993; 150:2019-2014. [PubMed: 7693985]

22. Kurhanewicz J, Vigneron DB, Nelson SJ, Hricak HJ, MacDonald JM, Konety B, Narayan P. Citrate as an in vivo marker to discriminate prostate cancer from benign prostatic hyperplasia and normal prostate peripheral zone: detection via localized proton spectroscopy. Urology. 1995; 45:459-467. [PubMed: 7533458]

23. Kurhanewicz J, Vigneron DB, Hricak H, Narayan P, Carrol P, Nelson SJ. Three dimensional hydrogen-1 MR spectroscopic imaging of the in situ human prostate with high spatial resolution. Radiology. 1996; 198:795-805. [PubMed: 8628874]

24. Heerschap A, Jager GJ, Van Der Graff M, Barentsz JO, De La Rosette JJMCH, Oosterhof GON, Ruijter ETG, Ruijs SHJ. In vivo proton MR spectroscopy reveals altered metabolite content in malignant prostate tissue. Anticancer Res. 1997; 17:1455-1460. [PubMed: 9179183]

25. Kaji Y, Kurhanewicz J, Hricak H, Sokolov DL, Huang LR, Nelson SJ, Vigneron DB. Localizing prostate cancer in the presence of postbiopsy changes in MR images: role of proton MR spectroscopic imaging. Radiology. 1998; 296:785-790. [PubMed: 9494502]

26. Liney GP, Turnbull LW, Lowry M, Turnbull LS, Knowles AJ, Horsman A. In vivo quantification of citrate concentration and water T2 relaxation time of the pathologic prostate gland using $1 \mathrm{H}$ MRS and MRI. Magn Reson Imaging. 1997; 15:1177-1186. [PubMed: 9408138]

27. Costello LC, Franklin RB. Bioenergetic theory of prostate malignancy. Prostate. 1994; 25:162166. [PubMed: 7520580]

28. Schiebler ML, Miyamoto KK, White M, Maygarden SJ, Mohler JL. In vitro high resolution 1Hspectroscopy of the human prostate: benign prostatic hyperplasia, normal peripheral zone and adenocarcinoma. Magn Reson Med. 1993; 29:285-291. [PubMed: 7680746]

29. Lowry M, Liney GP, Turnbull LW, Manton DJ, Blackband SJ, Horsman A. Quantification of citrate concentration in the prostate by proton magnetic resonance spectroscopy: zonal and agerelated differences. Magn Reson Med. 1996; 36:352-358. [PubMed: 8875404]

30. Price D. Comparative aspects of development and structure in the prostate. NCI Monogr. 1963; 12:1-27.

31. McNeal JE. Anatomy of the prostate. Prostate. 1980; 1:3-13. [PubMed: 7024953]

32. Parivar F, Hricak H, Shinhara K, Kurhanewicz J, Vigneron DB, Nelson SJ, Carroll PR. Detection of locally recurrent prostate cancer after cryosurgery: evaluation by transrectal ultrasound, magnetic resonance imaging, and three-dimensional proton magnetic resonance spectroscopy. Adult Urol. 1996; 48:594-599.

33. Gonic P, Oberleas D, Knechtges T, Prasad AS. Atomic absorption determination of zinc in the prostate. Invest Urol. 1969; 6:345-347. [PubMed: 5773519]

34. Habib FK, Mason MK, Smith PH, Stitch SR. Cancer of the prostate: early diagnosis by zinc and hormone analysis. Br J Cancer. 1979; 39:700-704. [PubMed: 87214] 


\section{SUMMARY}

1. It is now well-established that PCa is characterized by a dramatic decrease in prostate citrate levels.

2. Endorectal MRS detection of prostate citrate changes provides a noninvasive, sensitive, and accurate procedure for detecting $\mathrm{PCa}$, for localizing malignancy and determining its volume, and for monitoring the progression and regression of malignancy. The procedure provides a mapping of the prostate as a permanent record for the follow-up of patients.

3. The metabolic transformation of citrate-producing nonmalignant cells to citrateoxidizing malignant cells occurs early (probably as a premalignant stage), which permits the identification of PCa prior to histopathological identification.

4. In situ determination of citrate levels confirms that the glandular epithelial cells of the peripheral zone, and not of the central zone, are principally involved in the function of high citrate production and secretion.

5. In situ determination of citrate levels confirms that glandular BPH involves the proliferation and invasion of the central zone by citrate-producing epithelial cells.

6. In situ MRS measurements of citrate levels confirm the proposal that prostate malignancy involves a metabolic transformation in the citrate-related metabolism of the epithelial cells.

7. These relationships warrant immediate and intense study which will lead to the use of MRS as a diagnostic procedure in PCa; lead to the elucidation of the pathogenesis of prostate malignancy; and provide a metabolic basis for the development of new treatments against PCa. 

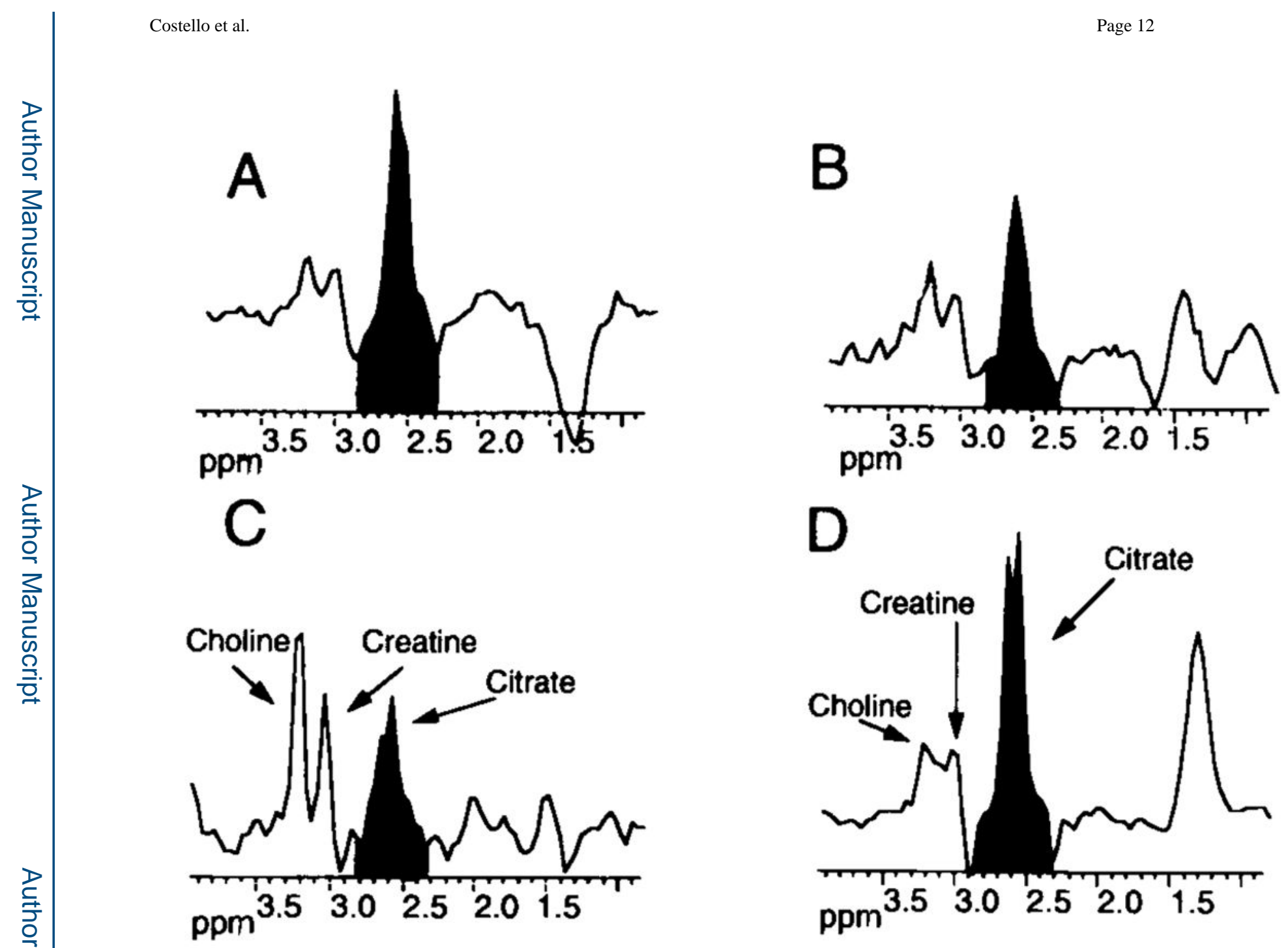

Fig. 1.

Representative in situ 1H MRS spectral analysis of the prostate of a subject with BPH and PCa. The analysis was achieved by 3-D MRS coupled with MRI ( $0.24 \mathrm{ccm}$ resolution).

Shaded area represents citrate signal. A: Region of the central zone which exhibited predominantly glandular BPH with some stromal BPH. B: Region of the central zone which exhibited predominantly stromal BPH with some glandular BPH. C: Region of the peripheral zone which exhibited prostate cancer. D: Region of the normal peripheral zone. Note the low citrate signal in the malignant peripheral zone compared to the normal peripheral zone, and the apparent differences in the citrate/choline + creatine ratios. Note that glandular BPH exhibits a high citrate level, whereas normal central zone typically exhibits very low citrate levels (not shown). As stroma replaces the glandular BPH, the citrate level decreases (Modified from Kurhanewicz et al. [23], with permission). 
A

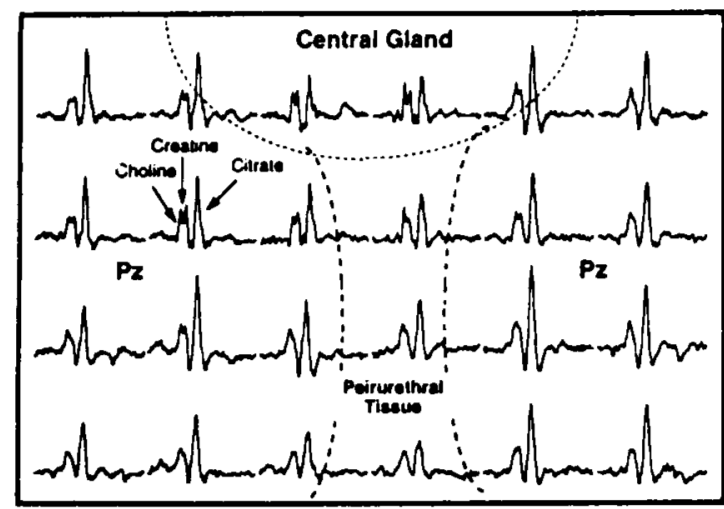

B

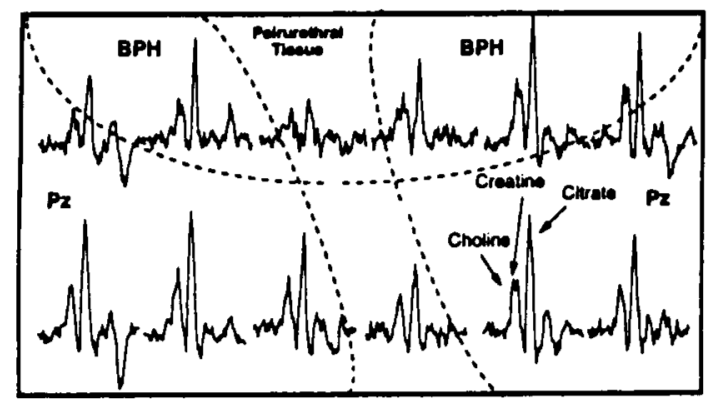

C

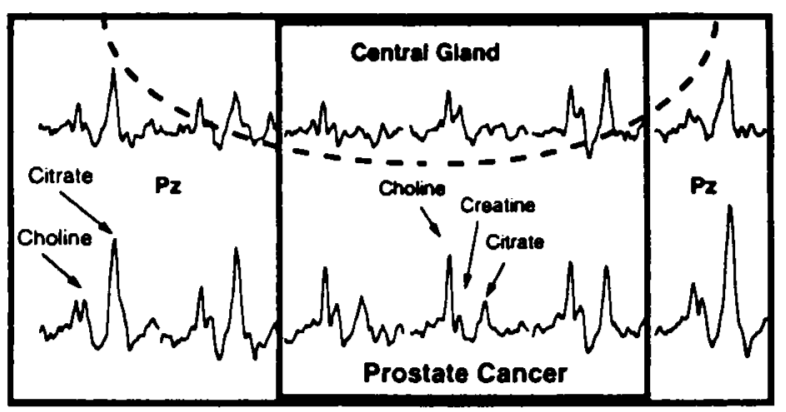

Fig. 2.

Typical contiguous mapping of in situ 1H MRS spectra of citrate relationships in normal and malignant prostate. A: Normal zonal differences in citrate. Note that the peripheral zone is characterized by a distinctly high, sharp peak referenced against the choline/creatine peak, whereas the central zone and periurethral regions exhibit a lower, less defined citrate signal.

B: Note that in BPH, the central zone exhibits a strong citrate signal comparable to the peripheral zone, whereas the periurethral region continues to exhibit a low citrate signal. This reveals that glandular BPH results from the invasion and proliferation of citrateproducing epithelial cells. $\mathbf{C}$ : Note that in $\mathrm{PCa}$, the malignant regions of the peripheral zone exhibit a greatly decreased citrate signal which is much lower than the choline signal. The normal regions of the peripheral zone exhibit the typical high citrate signal (compiled from figures in Kurhanewicz et al. [23], with permission). 


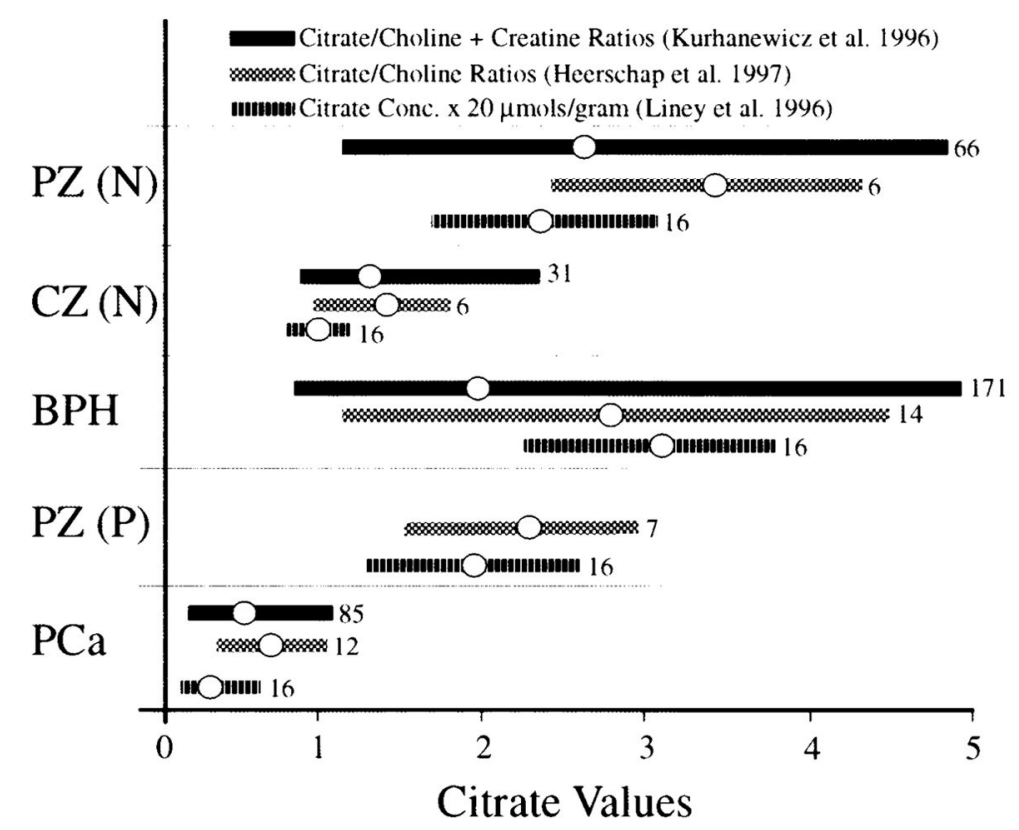

Fig. 3.

In situ 1H MRS measurements of prostate citrate levels and the detection of malignancy in humans. A composite of three independent reports. The data present the range of values obtained and the mean of the values. The $n$ for each set of measurements is presented at the end of each bar. PZ (N), normal peripheral zone of normal subject; PZ (P), normal peripheral zone of patients; $\mathrm{CZ}(\mathrm{N})$, normal central zone; $\mathrm{BPH}$, central zone with glandular and stromal hyperplasia; Pca, cancer in the peripheral zone (figure compiled from Kurhanewicz et al. [23], Heerschap et al. [24], and Liney et al. [26], with permission; data from Kurhanewicz et al. [23], originally presented as choline + creatine/citrate ratios, have been inverted for comparative purposes to be consistent with the presentation of the other two studies). 


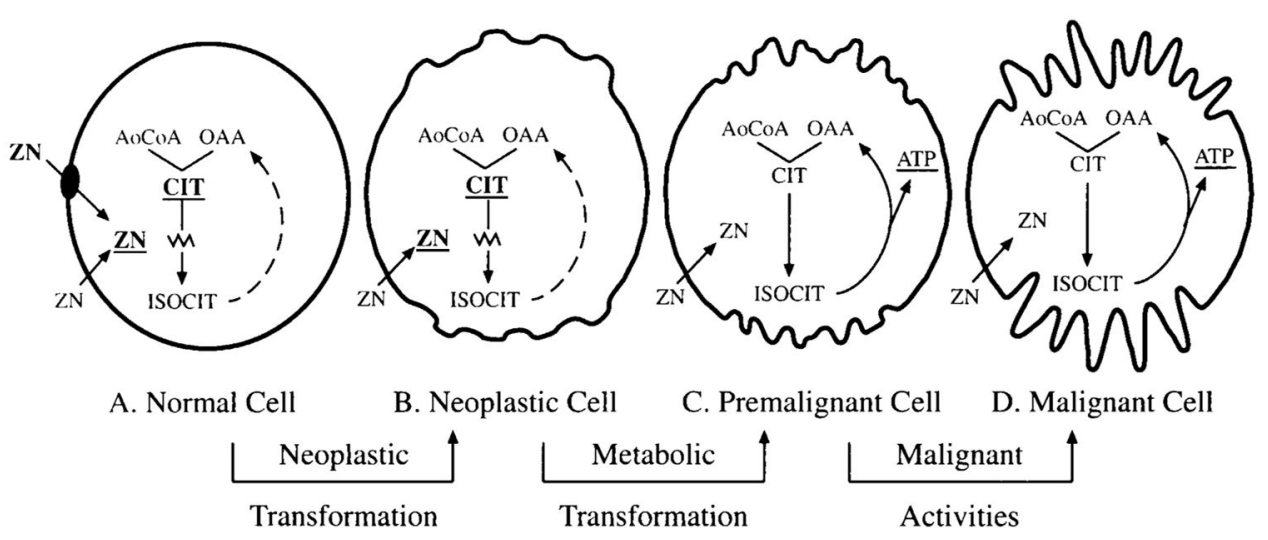

Fig. 4.

Proposed relationship of citrate in the development and progression of prostate malignancy as the basis for the diagnosis of PCa. A: Normal prostate glandular epithelial cells are citrate-producing cells in which high zinc accumulation inhibits citrate oxidation. These cells contain a zinc transport mechanism which is responsible for the intracellular accumulation of zinc. B: The neoplastic cell loses the ability to accumulate zinc, probably due to the lost activity of the zinc transporter. The inability to accumulate zinc causes a decline in intracellular zinc level, which results in removal of the zinc inhibition of citrate oxidation. C: Net citrate production no longer occurs, and the neoplastic cell is now a premalignant, citrate-oxidizing cell. D: Increased ATP production coupled to citrate oxidation occurs, and the premalignant cell is now able to perform its malignant activities and is histopathologically identifiable. This scheme accounts for the identification of early changes in zinc and citrate as a premalignant stage (C) prior to the appearance of detectable histopathological evidence of malignancy. 


\section{TABLE 1}

Representative Citrate Levels in the Human Prostate*

\begin{tabular}{lc}
\hline Normal central zone & 5,000 \\
Normal peripheral zone & 13,000 \\
BPH (mixed tissue) & $10,000-15,000$ \\
BPH (glandular tissue) & $20,000-50,000$ \\
PCa (mixed tissue) & $1,000-3,000$ \\
Malignant tissue & $<500$ \\
Stromal tissue & $150-300$ \\
Other soft tissues & $150-450$ \\
Blood plasma & $90-110$ \\
Prostatic fluid & $40,000-150,000$ \\
\hline
\end{tabular}

* All values are nmol/g wet weight. Values are derived from our own studies (L.C.C. and R.B.F.), and estimates from Liney et al. [26] and Lowry et al. [29]. 